\title{
The Temporalis Muscle Flap for Palate Reconstruction: Case Series and Review of the Literature
}

\author{
Tara Brennan ${ }^{1}$ Tristan M. Tham² Peter Costantino ${ }^{2}$ \\ ${ }^{1}$ Department of Otolaryngology, University of New Mexico, \\ Albuquerque, New Mexico, United States \\ 2 Department of Otolaryngology, New York Head and Neck Institute, \\ New York City, New York, United States \\ Address for correspondence Tara Brennan, MD. Assistant Professor, \\ Facial Plastic \& Reconstructive Surgery, Otolaryngology-Head \& Neck \\ Surgery, Department of Surgery, University of New Mexico, \\ Albuquerque, New Mexico \\ (e-mail: tristantham@gmail.com; ttham@northwell.edu).
}

Int Arch Otorhinolaryngol 2017;21:259-264.

\begin{abstract}
Introduction The temporalis myofascial (TM) is an important reconstructive flap in palate reconstruction. Past studies have shown the temporalis myofascial flap to be safe as well as effective. Free flap reconstruction of palate defects is also a popular method used by contemporary surgeons. We aim to reaffirm the temporalis myofascial flap as a viable alternative to free flaps for palate reconstruction.

Objective We report our results using the temporalis flap for palate reconstruction in one of the largest case series reported. Our literature review is the first to describe complication rates of palate reconstruction using the TM flap.

Methods Retrospective chart review and review of the literature.

Results Fifteen patients underwent palate reconstruction with the TM flap. There were no cases of facial nerve injury. Five (33\%) of these patients underwent secondary cranioplasty to address temporal hollowing after the TM flap. Three out of fifteen (20\%) had flap related complications. Fourteen (93\%) of the palate defects were successfully reconstructed, with the remaining case pending a secondary procedure to close the defect. Ultimately, all of the flaps $(100 \%)$ survived.

Keywords

- reconstructive surgical procedures

- palate

- temporal muscle

Conclusion The TM flap is a viable method of palate defect closure with a high defect closure rate and flap survival rate. TM flaps are versatile in repairing palate defects of all sizes, in all regions of the palate. Cosmetic deformity created from TM flap harvest may be addressed using cranioplasty implant placement, either primarily or during a second stage procedure.
\end{abstract}

\section{Introduction}

Palate defects caused by tumor resection or other pathologies substantially affect speech and swallowing. Leaving the defect open is suboptimal because it results in hypernasal speech and nasal regurgitation of ingested foods and liquids, among other issues. Risk of aspiration is also increased. Anterior palate defects, which include the maxilla and teeth, may also have readily discernible cosmetic effects.

received

November 18, 2016

accepted

December 21, 2016

published online

February 17, 2017

10.1055/s-0037-1598653. ISSN 1809-9777.
Before the advent and popularization of microvascular free tissue transfer, palate defect reconstruction was largely performed using local or regional tissue transfer. For larger defects, where local tissue transfer/rearrangement provided insufficient tissue for closure, regional flaps or obturators were used. Currently, free flap reconstruction using Anterolateral Thigh (ALT) or Radial Forearm Free Flap (RFFF) are popular options in many centers with trained microvascular

Copyright $\odot 2017$ by Thieme Revinter Publicações Ltda, Rio de Janeiro, Brazil

License terms

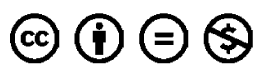


surgeons. Reconstruction involving free flap harvest at a distant tissue site and inset in the palate adds time and an additional surgical site to the procedure, and often involves two teams.

Reconstruction of palate defects with a temporalis myofascial (TM) flap is a relatively straightforward procedure. It is a regional flap in close proximity to the defect site, and may be performed by a single surgeon without microvascular training. It is effective for most patients, and good outcomes have been demonstrated in large case series. ${ }^{1}$ After TM flap harvest and inset, a hollowing in the temple is created. This secondary cosmetic side effect, however, can be treated with a cranioplasty, either as a secondary outpatient procedure or at the time of reconstructive surgery if desired by the patient. The surgeon may perform augmentation in the temporal fossa where the TM flap is harvested with a variety of materials, including autologous fat, injectable hydroxyapatite bone substitute, or preformed silicone implants, to name a few options. Other potential complications include alopecia around the incision line, trismus, fistula, and facial nerve paralysis, but they are not common. For these reasons, the TM flap can be considered by reconstructive surgeons as a reliable option for palate reconstruction, particularly when microvascular reconstruction is not readily available.

Patients that have undergone palate reconstruction using the TM flap are a heterogeneous group, with many different pathologies responsible for the defect. As a result, data from these procedures are mostly limited to case series and case reports. Outcomes data in recent large case series, however, have been positive and have shown good functional results among palate reconstructions performed using the temporalis muscle flap. ${ }^{1}$

The aims of this paper are to document the utility of and our outcomes with reconstruction of the palate using TM flaps in one of the largest case series to date. We also report the rate of postoperative cranioplasties performed to address the acquired cosmetic defect from TM flaps, of which there is little data. Our literature review is also the first to investigate the rates of flaprelated complications using the TM flap in palate reconstruction.

\section{Study Design/Methods}

We present a case series from the senior author's experience over a six-year period reconstructing hard and soft palate defects. This study was conducted with a waiver of oversight from the Institutional Review Board of Northwell Health System. Data collection was in compliance with the Health Insurance Portability and Accountability Act (HIPAA). We collected data on demographics, pathological features of disease, size of reconstruction, outcome measurements, and complications. Patients were included in this case series if they had a TM flap reconstruction of a hard and/or soft palate defect. There were 15 cases in this study, with a mean age range of 58 years (42-84 year range) (-Table 1 ).

We took defect sizes directly from operative reports. If the defect size was not mentioned in the report, we used pathology specimen descriptions and the operative report descriptions to estimate the size of the defect. As a reference for palate size, we used typical palate measurements from pre- viously compiled palate size databases. ${ }^{2,3}$ The palate defects were further categorized by location: A for the premaxilla, B for the hard palate, and $C$ for the soft palate.

We performed a literature review to identify studies that examined the use of TM/TPF flaps for palate reconstruction. A MEDLINE database search via the PubMed.gov portal was performed using the following terms: temporalis flap palate, temporalis muscle flap palate, temporoparietal fascia flap palate, temporalis palate reconstruction, temporalis palate, temporalis muscle hard palate reconstruction, and temporalis flap palatal reconstruction. Single case reports were excluded. Papers that did not specifically address palate reconstruction were excluded. We identified thirteen case series published between 1991 and 2012 on TM flaps specifically for palate reconstruction (-Table 2 ). Data recorded in the literature review were the numbers of patients and type and incidence of complications.

\section{Results}

This retrospective study included 15 patients, with a mean age range of 58 years (42-84 year range). A wide mix of palate pathologies - benign, malignant, and others - were responsible for the palate defects that presented for reconstruction: the most commonly represented in this series was adenoid cystic carcinoma (ACC), which was involved in 5 of the 15 cases (33\%). The rest are summarized in - Table $\mathbf{1}$. Size of the defects reconstructed ranged from a maximum diameter of 1 to $6 \mathrm{~cm}$, with a mean maximum diameter $3.5 \mathrm{~cm}$. Defects were unilateral or bilateral and categorized by location ( A - premaxilla, $\mathrm{B}$ - hard palate, $C$ - soft palate) within the palate. We used TM flaps to repair defects in all regions $(A, B, C)$ of the palate.

The complications are summarized in - Table 1. Three out of fifteen patients (20\%) had flap related complications. The TM complication rate was similar to free flap complication rates published. ${ }^{4}$ Two patients had residual fistula (14\%) and one had trismus (7\%). Fifteen of the flaps (100\%) survived.

All flap related complications, with one exception, were successfully rectified with subsequent minor surgical procedures. The exception is one patient with a residual fistula whom at the time of this publication was being scheduled for corrective surgery.

Ultimately, all flaps (100\%) survived, and 14 out of 15 patients (93\%) had defects successfully reconstructed with TM flaps.

There were no cases of facial nerve paresis. Five (33\%) of the $15 \mathrm{TM}$ flap patients elected to pursue aesthetic correction of temporal hollowing with cranioplasty. These patients underwent subsequent temporal cranioplasty using either autologous fat or injectable hydroxyapatite (HA) bone substitute. Lastly, none of the patients self-reported any long term difficulties with speech or swallowing function.

\section{Discussion}

The TM flap has, despite the advent of microsurgical techniques, remained a viable alternative in palate defect repair because of several factors. First, it is a hearty and well 
Table 1 Clinical data of patients with reconstruction with temporalis muscle flap

\begin{tabular}{|l|l|l|l|l|l|l|l|l|}
\hline$\#$ & Age & Pathology & $\begin{array}{l}\text { Type of } \\
\text { Flap }\end{array}$ & Region & $\begin{array}{l}\text { Size } \\
(\mathbf{c m})\end{array}$ & $\begin{array}{l}\text { Flap related } \\
\text { complications }\end{array}$ & $\begin{array}{l}\text { Need for } \\
\text { flap revision }\end{array}$ & Cranioplasty \\
\hline 1 & 46 & Recurrent Ameloblastoma & TM & A/B & 3 & No & No & Yes \\
\hline 2 & 84 & SCC & TM & B & 4.5 & No & No & No \\
\hline 3 & 61 & ACC & TM & A/B & 3 & No & No & No \\
\hline 4 & 68 & SCC & TM & B/C & 4 & No & No & No \\
\hline 5 & 46 & ACC & TM & A/B & 3 & Residual fistula & Yes & No \\
\hline 6 & 47 & Pleomorphic Adenoma & TM & A/B/C & 6 & No & No & No \\
\hline 7 & 51 & ACC & TM & B & 3 & No & No & Yes \\
\hline 8 & 42 & $\begin{array}{l}\text { Mucoepidermoid } \\
\text { Carcinoma }\end{array}$ & TM & B/C & 3.5 & No & No & Yes \\
\hline 9 & 64 & $\begin{array}{l}\text { Defect secondary } \\
\text { to chronic cocaine abuse }\end{array}$ & TM & $\begin{array}{l}\text { B/C, } \\
\text { part of A }\end{array}$ & 3.5 & No & No & Yes \\
\hline 10 & 50 & Adenocarcinoma & TM & B/C & 3 & Residual fistula* & Yes & No \\
\hline 11 & 68 & ACC & TM & B & 3 & Trismus & No & Yes \\
\hline 12 & 81 & $\begin{array}{l}\text { Defect Secondary to } \\
\text { latrogenic Osteoradionecrosis }\end{array}$ & TM & b/l A/B/C & 4 & No & No & No \\
\hline 13 & 47 & ACC & TM & B & 1 & No & No & No \\
\hline 14 & 75 & Malignant Melanoma & TM & A/B & 3 & No & No & No \\
\hline 15 & 45 & Adenocarcinoma & TM & A/B/C & 4 & No & No & No \\
\hline
\end{tabular}

Abbreviations: ACC, adenoid cystic carcinoma; b/I, bilateral; SCC, Squamous cell carcinoma; TF, temporoparietal fascia; TM, temporalis muscle. ${ }^{*}$ corrected with surgery, ${ }^{* *}$ size of defect, diameter $(\mathrm{cm})$.

Region A - premaxilla, Region B - hard palate, Region C - soft palate.

vascularized flap with low rates of necrosis. Next, it is large enough to fill large defects, even bilateral palate defects. It offers good functional outcomes for speech and swallowing. Also, the incision used to harvest this flap gives the surgeon access to additional tissue for reconstruction, including the TPF, periosteum over the bone of the temporal fossa, and calvarial bone. Finally, a single surgeon can perform a resection and the reconstruction in a single operative field.

The TM flap is a robust, well vascularized flap with excellent healing and functional outcomes. Large case series using the flap have consistently showed low rates of flap failure. Our study is the third largest case series to date on TM flap reconstruction of palate defects, and also examines secondary cranioplasty data. Our literature review looked at complication rates of TM flaps used to manage palate defects specifically. In the patient population identified in the literature review, 1 patient out of 106 recorded had flap necrosis $(\sim 1 \%)$, and the flap survived after local surgical debridement (-Table 2). The data in our case series is in line with the existing literature; the incidence of partial flap necrosis was low at one out of twenty patients (5\%), with flap survival after local surgical debridement. Of note, our patient with partial TM flap necrosis had a background history of Wegner's granulomatosis, which caused the palatal defect. This could possibly have predisposed the patient to having a compromised vascular supply and therefore flap necrosis (-Table $\mathbf{1}$ ).

Three out of fifteen (20\%) had flap related complications. The complications were residual fistula ( 2 patients) and trismus (1 patient). All flap related defects, with one exception, were successfully rectified with a subsequent minor surgical procedure in the OR. The patient with trismus was managed with a Brisemont procedure. One patient with a post-operative fistula is pending corrective surgery at the time of this publication. Ultimately, all 14 out of 15 patients (93\%) had defects successfully reconstructed with TM flaps, and all the flaps (100\%) survived.

There were no cases of facial nerve compromise, which is lower than the reported facial nerve complication rate in the literature, which has been reported to be $19.2 \%$ and $2.7 \%$ for transient paresis and permanent paralysis, respectively. ${ }^{5}$

The TM flap offers a substantial amount of tissue to fill large bilateral palate defects. In our case series, the TM flap was used to fill unilateral defects up to $6 \mathrm{~cm}$ in length and bilateral defects up to $4 \mathrm{~cm}$ encompassing all regions of the palate (pre-maxilla with hard and soft palate). Similarly, Abubaker and Abouzgia reported closing palate defects up to $6 \times 5 \mathrm{~cm}$ in size encompassing both the hard and soft palate, with no complications. ${ }^{6}$ Guidelines on the maximum size of palate defects that can be closed with the TM flap have not been well established. Considerations that affect the maximum size of closure include the length and bulk of the temporalis muscle, as well as patient-specific anatomy during the flap rotation and tunneling into the oral cavity. Guidelines proposed by Brown and Shaw ${ }^{7}$ describe that the TM flap can be used for: vertical maxillary defects, as long as they do not encompass orbits/nasal bones; horizontal palatal defects less 
Table 2 Summary of the most commonly reported complications associated with the use of TM/TF flaps specifically for palate reconstruction

\begin{tabular}{|l|l|l|l|}
\hline$\#$ & Paper & Number of Patients & Complications/notes \\
\hline 1 & Browne $^{1}$ & 72 & $\begin{array}{l}3(4.2 \%) \text { Trismus, 4 (5.6\%) Choanal stenosis, (1.4\%) Ocular } \\
\text { muscle entrapment, 21 (29.2\%) Serous otitis media, 10 (13.9\%) } \\
\text { Temporal fossa infection. 31 (43\%) patients had at least 1 complication }\end{array}$ \\
\hline 2 & Krzymański $^{18}$ & 3 & $2(66 \%)$ oronasal fistula, managed with secondary suture \\
\hline 3 & Abu-El Naaj $^{19}$ & 5 & $1(20 \%)$ necrotic flap, resolved after local debridement \\
\hline 4 & Wong $^{20}$ & 6 & $\begin{array}{l}1(16.7 \%) \text { Cheek Fullness, 1 (16.7\%) Cheek Depression, 2 (33.3\%) } \\
\text { Velopharyngeal Incompetance, 2 (33.3\%) Siaolocele. }\end{array}$ \\
\hline 5 & Abubaker $^{6}$ & 8 & No complications \\
\hline 6 & Hanasono $^{21}$ & 2 & No complications \\
\hline 7 & Thomson $^{22}$ & 4 & $*$ \\
\hline 8 & Cordeiro $^{23}$ & 24 & $*$ \\
\hline 9 & Clauser $^{5}$ & 3 & $*$ \\
\hline 10 & Del Hoyo $^{24}$ & 7 & $*$ \\
\hline 11 & Van der Wal $^{25}$ & 4 CLP & No complications \\
\hline 12 & Colmenero $^{26}$ & 4 & 3 (75\%) Trismus. Rest of complications not site specific \\
\hline 13 & Tartan $^{27}$ & 2 adult CLP & No complications \\
\hline
\end{tabular}

Abbreviations: CLP, cleft lip and palate.

* complications not listed as specific to the palate.

than or equal to one-half the width of the palate; or a combination of the two. ${ }^{7}$ This set of guidelines may be considered slightly conservative, as our case series and other authors $^{1,6}$ have shown successful repair with significantly larger bilateral palate defects encompassing premaxilla, hard palate, and soft palate.

The versatility of the TM flap is also due to the availability of many tissue types (fascia, muscle, periosteum, skin, and calvarial bone) available to the surgeon to reconstruct the patient-specific defect.

Palate defect reconstruction aims to achieve some form of pre-disease functional restoration, of which speech and swallowing are important contributors to the patient's quality of life. We report no incidence of speech or swallowing defects in our case series. However, this was subjective and self-reported; as per standard management, formal testing with validated measurements would only have been done in patients reporting or have been clinically assessed to have functional deficiencies. The literature shows good functional outcomes in palate reconstruction with the TM flap - Browne et al assessed the post-operative function of 27 patients with TM flaps and found that mean nasalance (defined as degree of velopharyngeal opening in voiced speech) were within normal limits for connected speech tasks. Furthermore, swallowing scores in this study measured via MDADI (MD Anderson Dysphagia Inventory score) ranged from good to mildly affected. ${ }^{1}$ In a smaller case series of 9 patients, Dallan and colleagues assessed swallowing function after TM flap reconstruction, with all but two patients having good swallowing function at more than 6 months post-operatively. ${ }^{8}$

Functional outcomes in microvascular free flap reconstruction are also good. Similar to TM reconstruction, swallowing function after reconstruction with a radial forearm free flap (RFFF) is excellent, with most patients eventually resuming normal diets. ${ }^{9}$ Both TM and RFFF methods of reconstruction have superior swallowing function compared with organ preservation strategies, such as chemoradiation treatment regimens. $^{10,11}$

There is some evidence that speech outcomes may be better among patients reconstructed with TM flaps rather than RFFF, particularly among patients with large soft palate defects. Seikaly et al examined speech and swallowing function in 18 patients with RFFF reconstruction, and found that mean nasalance scores were higher than normal values (34\% SD20, pre-radiotherapy; 31\% SD15, post-radiotherapy) in RFFF patients with defects larger than half the soft palate. ${ }^{9}$ In contrast, Browne et al found that mean nasalance scores for patients with TM reconstruction were at normal values for consecutive speech-low pressure (21\% SD10), consecutive speech-high pressure (17\% SD10), and sustained vowel tasks (26\% SD17), regardless of defect size and location. ${ }^{1}$ It is interesting to note that the location and size of the palate defect had a bearing on the eventual functional speech outcomes in RFFF reconstruction, as the study by Seikaly et al shows worse speech outcomes in patients with defects larger than half the soft palate. The study by Browne et al showed that nasalance scores did not appear to vary according to defect size/region, and remained universally lower than the RFFF data. These data point to the possibility that TM flap reconstruction could afford superior speech outcomes compared with RFFF, in particular nasalance. However, the aforementioned data are limited to small case series with relatively large standard deviations. 
TM flap reconstruction benefits from the donor and defect areas being in the same surgical site. Because of this, a single surgeon or surgical team is able to perform this reconstruction. Microvascular free flap reconstruction requires two operation fields, and would arguably be less suitable for certain groups of patients who have a higher risk of failure of vascular anastomoses (high cardiovascular risk, candidates for radiotherapy).

However, TM flap reconstruction of the palate comes with several drawbacks. One of the main sequelae from TM flap reconstruction is the significant abnormal aesthetic of temporal hollowing, especially when the full bulk of the temporalis muscle is used. This is remedied with a secondary cranioplasty to restore aesthetic symmetry, as described above. In our case series, only patients with larger deficits elected for cranioplasties.

Another significant limitation of TM flaps, unlike microvascular flaps, is that TM flap reconstruction precludes any form of osseointegrated dental devices. ${ }^{12}$ This puts a limitation in the locations that the TM flap would be able to repair to restore normal mechanical digestion and aesthetics, for example in the anterior pre-maxilla.

Despite good outcomes and reliability of the TM flap, current trends seem to favor microvascular transfer for primary palatal reconstruction over regional flaps. ${ }^{13-17}$ The reasons for preference of obturation/prosthodontic or free flap microvascular transfer over regional flap options are varied. Using obturators and opting to leave an open cavity to monitor the tumor bed is one of the main reasons for not choosing surgical reconstruction. However, patients experience difficulties with these devices with discomfort, as well as lifestyle changes to accommodate deglutition and communication.

Free flap microvascular reconstruction using ALT or RFFF are currently popular options for palate reconstruction. The reason for their increasing use over regional reconstructive options likely stems, at least in part, due to contemporary surgeons' increasing comfort and expertise in microsurgical techniques. Additionally, as regional flap reconstruction for palate defects becomes less popular, familiarity with surgical technique for harvesting them has decreased. Opting for free flap reconstruction over regional/TM flap reconstruction for palate defects removes risk of injury to the frontal branch of the facial nerve and need for adjuvant procedures to address acquired temporal hollowing. Furthermore, the aesthetic defect made with an ALT or RFFF may be considered less noticeable than that of a TM flap. Microvascular techniques also offer the possibility of osseous integration for dental implants. However, opting for microvascular reconstruction may add time to the procedure, as well as require an additional surgical team to harvest the donor tissue. The TM flap offers comparable outcomes with microvascular techniques in terms of complications, and the data suggests it may offer superior functional outcomes particularly in speech tasks. Whichever technique the reconstructive surgeon ultimately chooses, he will have to be cognizant of each method's various risks and benefits.

\section{Conclusion}

The TM flap is a viable method of palate defect closure using a single surgeon approach. The TM flap has a high defect closure rate and flap survival rate. TM flaps are versatile in repairing palate defects of all sizes, in all regions of the palate. Cosmetic deformity created from TM flap harvest may be addressed using cranioplasty implant placement, either primarily or during a second stage procedure. Our literature review is the first to describe complication rates of palate reconstruction using the TM flap.

\section{References}

1 Browne JD, Butler S, Rees C. Functional outcomes and suitability of the temporalis myofascial flap for palatal and maxillary reconstruction after oncologic resection. Laryngoscope 2011;121(06): 1149-1159

2 Ferrario VF, Sforza C, Schmitz JH, Colombo A. Quantitative description of the morphology of the human palate by a mathematical equation. Cleft Palate Craniofac J 1998;35(05):396-401

3 You M, Li X, Wang H, et al. Morphological variety of the soft palate in normal individuals: a digital cephalometric study. Dentomaxillofac Radiol 2008;37(06):344-349

4 Futran ND, Haller JR. Considerations for free-flap reconstruction of the hard palate. Arch Otolaryngol Head Neck Surg 1999;125(06): 665-669

5 Clauser L, Curioni C, Spanio S. The use of the temporalis muscle flap in facial and craniofacial reconstructive surgery. A review of 182 cases. J Craniomaxillofac Surg 1995;23(04):203-214

6 Abubaker AO, Abouzgia MB. The temporalis muscle flap in reconstruction of intraoral defects: an appraisal of the technique. Oral Surg Oral Med Oral Pathol Oral Radiol Endod 2002;94(01): 24-30

7 Brown JS, Shaw RJ. Reconstruction of the maxilla and midface: introducing a new classification. Lancet Oncol 2010;11(10): 1001-1008

8 Dallan I, Lenzi R, Sellari-Franceschini S, Tschabitscher M, Muscatello L. Temporalis myofascial flap in maxillary reconstruction: anatomical study and clinical application. J Craniomaxillofac Surg 2009;37(02):96-101

9 Seikaly H, Rieger J, Wolfaardt J, Moysa G, Harris J, Jha N. Functional outcomes after primary oropharyngeal cancer resection and reconstruction with the radial forearm free flap. Laryngoscope 2003;113(05):897-904

10 Smith RV, Kotz T, Beitler JJ, Wadler S. Long-term swallowing problems after organ preservation therapy with concomitant radiation therapy and intravenous hydroxyurea: initial results. Arch Otolaryngol Head Neck Surg 2000;126(03):384-389

11 Lazarus CL, Logemann JA, Pauloski BR, et al. Swallowing disorders in headand neckcancer patients treated withradiotherapyandadjuvant chemotherapy. Laryngoscope 1996;106(9 Pt 1):1157-1166

12 Futran ND, Mendez E. Developments in reconstruction of midface and maxilla. Lancet Oncol 2006;7(03):249-258

13 Bianchi B, Ferri A, Ferrari S, Copelli C, Sesenna E. Maxillary reconstruction using anterolateral thigh flap and bone grafts. Microsurgery 2009;29(06):430-436

14 Browne JD, Burke AJ. Benefits of routine maxillectomy and orbital reconstruction with the rectus abdominis free flap. Otolaryngol Head Neck Surg 1999;121(03):203-209

15 Davison SP, Sherris DA, Meland NB. An algorithm for maxillectomy defect reconstruction. Laryngoscope 1998;108(02):215-219

16 Johnson JT, Aramany MA, Myers EN. Palatal neoplasms: reconstruction considerations. Otolaryngol Clin North Am 1983;16(02): 441-456 
17 Wolff KD, Kesting M, Thurmüller P, Böckmann R, Hölzle F. The anterolateralthighasauniversaldonorsiteforsofttissuereconstructionin maxillofacialsurgery.JCraniomaxillofacSurg2006;34(06):323-331

18 Krzymański G, Dąbrowski J, Przybysz J, Domański W, Biernacka B, Piętka T. Temporal muscle flap in reconstruction of maxillo-facial tissues. Contemp Oncol (Pozn) 2012;16(03):244-249

19 Abu-El Naaj I, Leiser Y, Liberman R, Peled M. The use of the temporalis myofascial flap in oral cancer patients. J Oral Maxillofac Surg 2010;68(03):578-583

20 Wong TY, Chung $\mathrm{CH}$, Huang JS, Chen HA. The inverted temporalis muscle flap for intraoral reconstruction: its rationale and the results of its application. J Oral Maxillofac Surg 2004;62(06):667-675

21 Hanasono MM, Utley DS, Goode RL. The temporalis muscle flap for reconstruction after head and neck oncologic surgery. Laryngoscope 2001;111(10):1719-1725

22 Thomson CJ, Allison RS. The temporalis muscle flap in intraoral reconstruction. Aust N Z J Surg 1997;67(12):878-882
23 Cordeiro PG, Wolfe SA. The temporalis muscle flap revisited on its centennial: advantages, newer uses, and disadvantages. Plast Reconstr Surg 1996;98(06):980-987

24 Alonso del Hoyo J, Fernandez Sanroman J, Gil-Diez JL, Diaz Gonzalez FJ. The temporalis muscle flap: an evaluation and review of 38 cases. J Oral Maxillofac Surg 1994;52(02):143-147, discussion 147-148

25 van der Wal KG, Mulder JW. The temporal muscle flap for closure of large palatal defects in CLP patients. Int J Oral Maxillofac Surg 1992;21(01):3-5

26 Colmenero C, Martorell V, Colmenero B, Sierra I. Temporalis myofascial flap for maxillofacial reconstruction. J Oral Maxillofac Surg 1991;49(10):1067-1073

27 Tartan BF, Sotereanos GC, Patterson GT, Giuliani MJ. Use of the pharyngeal flap with temporalis muscle for reconstruction of the unrepaired adult palatal cleft: report of two cases. J Oral Maxillofac Surg 1991;49(04):422-425 